\title{
Correction to: Heat stress alters genome-wide profiles of circular RNAs in Arabidopsis
}

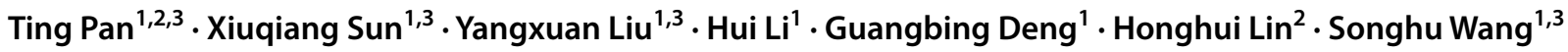

Published online: 22 January 2018

๑) Springer Science+Business Media B.V., part of Springer Nature 2018

\section{Correction to: Plant Molecular Biology \\ https://doi.org/10.1007/s11103-017-0684-7}

Due to an unfortunate turn of events, the first name of the fifth author appeared incorrectly in the original publication and should have read Guangbing.

The correct representation of the authors' names and their affiliation is listed here and should be treated as definitive.

The original article can be found online at https://doi.org/10.1007/ s11103-017-0684-7.

\section{Songhu Wang}

wangsh1@cib.ac.cn

1 CAS Center for Excellence in Molecular Plant Sciences, Chengdu Institute of Biology, Chinese Academy of Sciences, Chengdu 610041, China

2 Ministry of Education, Key Laboratory for Bio-Resource and Eco-Environment, College of Life Science, State Key Laboratory of Hydraulics and Mountain River Engineering, Sichuan University, Chengdu 610064, China

3 University of Chinese Academy of Sciences, Beijing 100049, China 